\title{
Southern Carbon Stars Found on Near-IR Objective-Prism Plates
}

\author{
D. JACK MacCONNELL \\ Computer Sciences Corp., Space Telescope Science Institute \\ Baltimore MD, U.S.A.
}

\begin{abstract}
A large set of deep objective-prism plates taken with the Curtis Schmidt telescope of The University of Michigan at Cerro Tololo, Chile is being used to search for cool supergiants and carbon stars along the southern galactic plane. The exposures are on hypersensitized I-N plates covering the $6800-8800 \AA$ region at a dispersion of $3400 \AA \mathrm{mm}^{-1}$ at the telluric A-band and reach a limiting magnitude of $I \approx 13.5$. The sky coverage extends to 6.5 on either side of the southern galactic plane, but not all fields have been searched to date. When not overexposed, cool carbon stars are readily distinguished by their prominent CN bands around $7900 \AA$ and can be detected to the plate limit. A few hundred new $\mathrm{C}$ stars found on these plates were published in 1988, and here we report additional $\mathrm{C}$ stars found in recently-surveyed regions covering about 600 square degrees; about one-third of those found are unpublished.
\end{abstract}

